\title{
Analysis of serum enzyme levels in a rabbit model of acute mesenteric ischemia
}

\author{
FU-XIAN ZHANG, BING-BING MA, GANG-ZHU LIANG and HUAN ZHANG
}

\author{
Department of Vascular Surgery, Shijitan Hospital, Capital Medical University, Beijing 100038, P.R. China
}

Received April 13, 2011; Accepted August 2, 2011

DOI: $10.3892 / \mathrm{mmr} .2011 .553$

\begin{abstract}
Early diagnosis of acute mesenteric ischemia (AMI) remains very difficult, partly due to the fact that useful markers of early small bowel ischemia have not yet been identified. Thus, in this study, we aimed to evaluate the levels of serum intracellular enzymes in the tissues and organs in a controlled animal model of mesenteric intestinal ischemia. Forty-eight New Zealand rabbits were divided into 4 groups including the control, artery ligation, vein ligation, and both artery and vein ligation groups. Plasma samples were obtained at 0-, 1-, 3-, 6- and 9-h time-points and enzyme levels were determined. The bowel color and vitality were observed at the same time. The bowel showed an appearance of infarction after a period of ischemia in the animals. Six and $9 \mathrm{~h}$ after superior mesenteric artery ischemia, alanine aminotransferase (ALT) and aspartate aminotransferase (AST) levels increased significantly. However, after $3 \mathrm{~h}$ of superior mesenteric venous (SMV) ligation, $\gamma$-glutamyl transpeptidase levels in the blood were considerably higher compared to the control group. Six hours after SMV ischemia, ALT, AST, alkaline phosphatase and lactate dehydrogenase levels were significantly elevated compared to those pre-ligation. Serum enzyme levels during intestinal ischemia are not able to provide sufficient information as regards the extent and reversibility of intestinal ischemia, although, they may be able to reflect the presence of injury.
\end{abstract}

\section{Introduction}

Acute mesenteric ischemia (AMI) is a life-threatening condition characterized by the sudden occlusion of mesenteric arteries followed by impairment of intestinal blood flow. It is classified as acute mesenteric arterial embolism and thrombosis, mesenteric venous thrombosis, and nonocclusive mesenteric ischemia (1-3). Early diagnosis of AMI remains very difficult, primarily because the early clinical symptoms

Correspondence to: Dr Fu-Xian Zhang, Department of Vascular Surgery, Shijitan Hospital, Capital Medical University, Beijing 100038, P.R. China

E-mail: fuxianvascular@gmail.com; fuxianvascular@yahoo.cn

Key words: intestinal ischemia, plasma enzyme levels, rabbits of this disease are non-specific and initially subtle. Therefore, diagnosis is considerably delayed, by which stage both the patient and the intestine are severely compromised. Despite the progress in the diagnosis and treatment of AMI over the past four decades, it still has a poor prognosis with an in-hospital mortality rate of 59-93\% (4-6).

To date, there are no reliable and easy-to-use laboratory markers available for the accurate and early diagnosis of ischemia. Although various markers for AMI have been investigated, most of them have shown low sensitivity and are not capable to be used in clinical practice.

In this study, we aimed to determine whether plasma enzyme levels would become elevated in rabbit models of AMI and whether these elevations could be used as a predictor for AMI.

\section{Materials and methods}

Animal model. Forty-eight male and female New Zealand white rabbits (2.5-3 kg body weight) were assigned into 4 groups (12 in each). Animals were handled with the approval and according to the rules of the animal research committee. All experimental procedures were performed in well equipped animal facilities. The animals were divided into the 4 following groups: group I (controls), animals underwent sham laparotomy with exposure of the superior mesenteric artery (SMA) and superior mesenteric venous (SMV) after appropriate anesthesia; group II, SMA and identified collaterals were ligated; group III, SMV and identified collaterals were ligated; and group IV, both SMA and SMV were ligated.

Surgical procedure. The animals were anesthetized with $3 \%$ barbital, 30-35 mg/intravenous injections. An internal femoral vein intravenous catheter was then placed so that blood samples could be taken from the animals.

The experimental animals underwent a standard midline laparotomy. The small intestine was reflected to the left of the abdominal incision and then the SMA and SMV were identified. Mesenteric ischemia was confirmed when the mesenteric pulsation was lost and the intestine became pale (Fig. 1).

After SMA or/and SMV ligation, heparinized blood samples were obtained from the femoral vein at 0 - (before ligation), 1-, 3-, 6- and 9-h time-points. At each time-point, plasma was separated immediately and stored at $-20^{\circ} \mathrm{C}$ until analysis. Plasma aspartate aminotransferase (AST), alanine amino- 

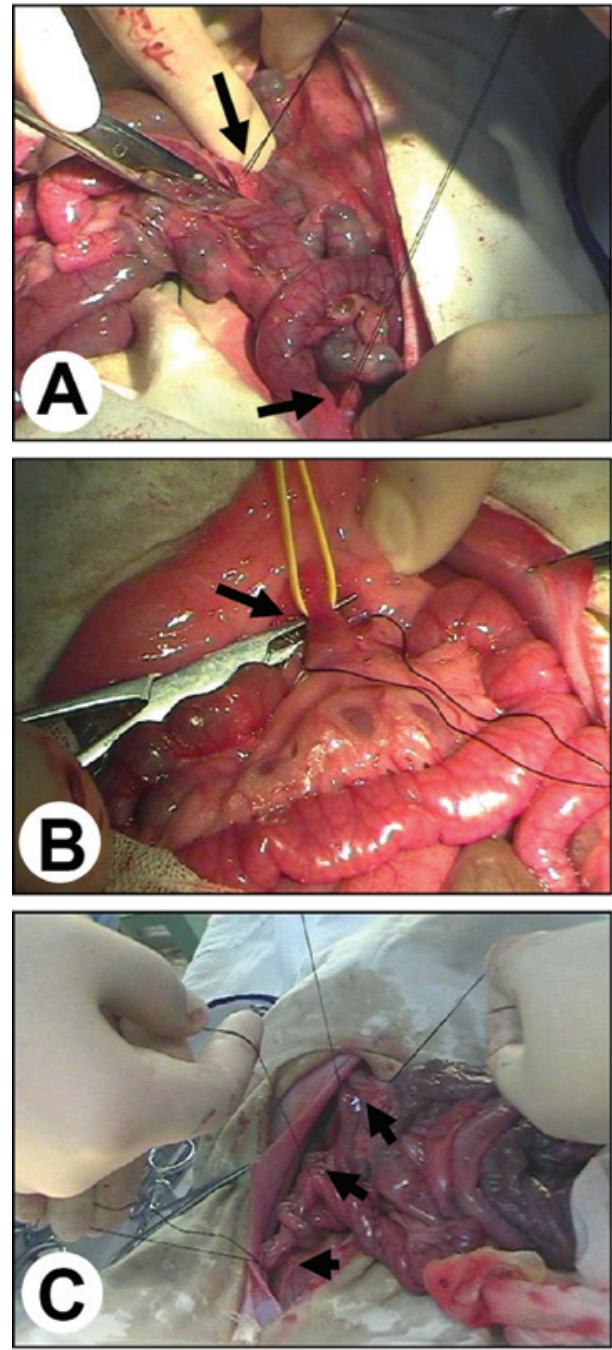

Figure 1. Surgical procedure using the animal model. (A) Superior mesenteric artery (SMA) ligation. (Black arrow, SMA). (B) Superior mesenteric venous (SMV) ligation. (Black arrow, SMV). (C) SMA and SMV ligation. (Black arrow, SMA and SMV).
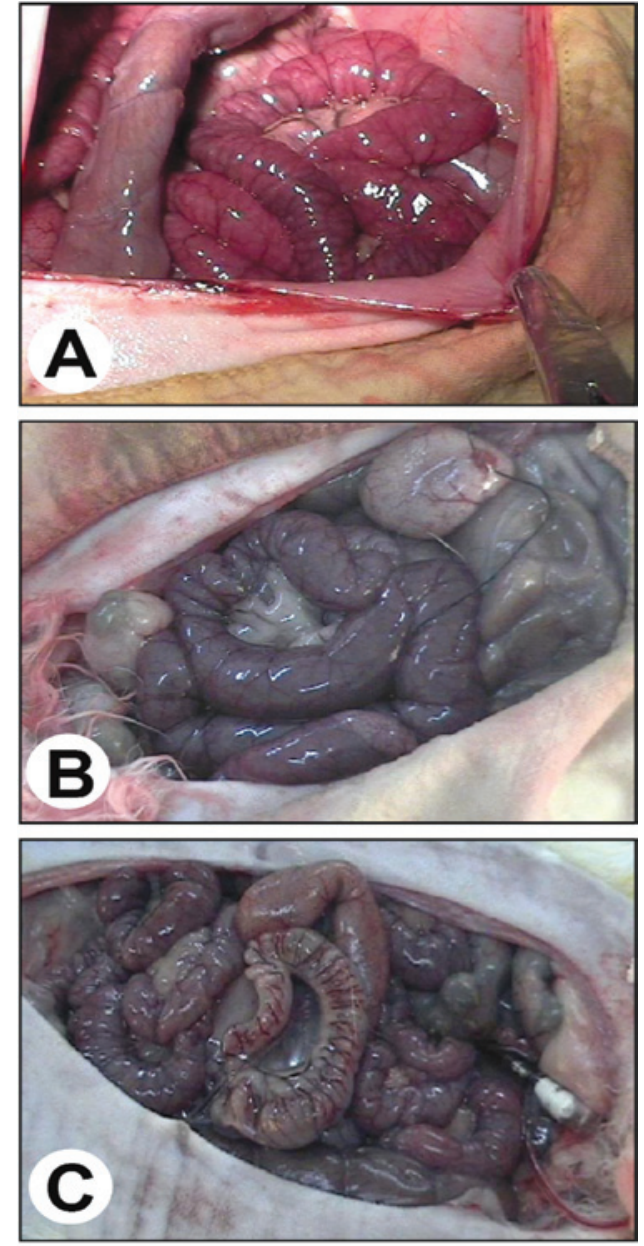

Figure 3. Bowel of the rabbits in the superior mesenteric venous (SMV) ligation group. (A) One hour after SMV ligation, changes in the bowel were evident, similar to those seen after $3 \mathrm{~h}$ in the superior mesenteric artery (SMA) ligation group. Necrosis and hemorrhage of the small bowel could be seen. (B) Three hours after SMV ligation, the bowel changed to a black color and extensive hemorrhagic infarction of the bowel was found. (C) Six hours after SMV ligation, the appearance of vitality was lost and most of the animals died.
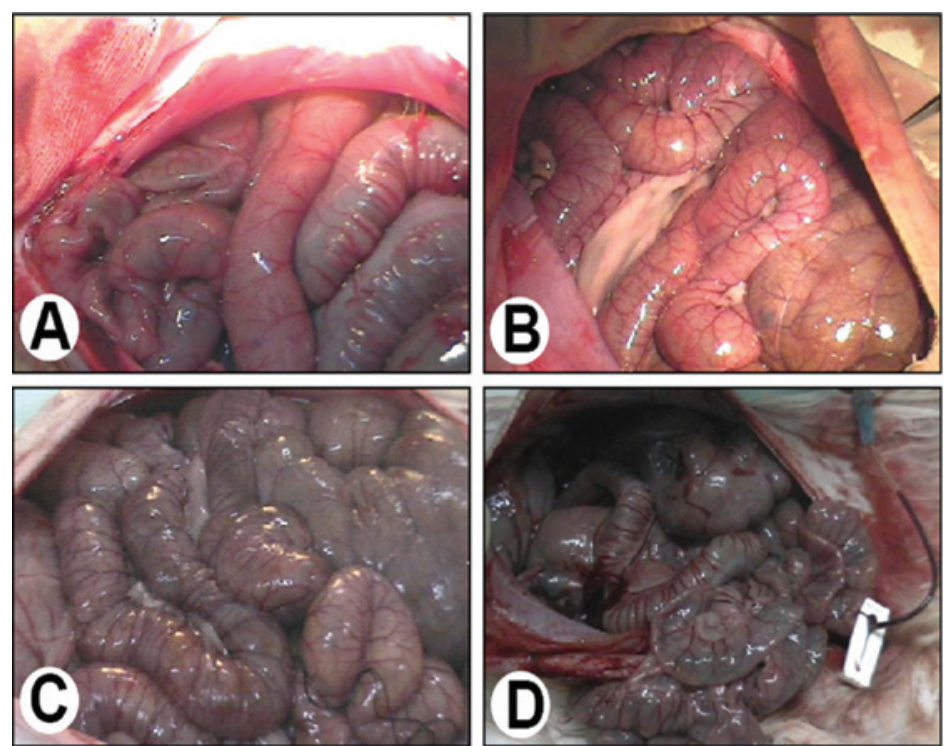

Figure 2. Bowel of the rabbits of the superior mesenteric artery (SMA) ligation group. (A) One hour after SMA ligation, the bowel became pale and dilated. (B) Three hours after ligation, the color of the bowel changed to grey and focal necrosis of the bowel could be seen. (C) Six hours after SMA ligation, extensive patchy necrosis and dilation throughout the small bowel with free hemorrhagic intraperitoneal fluid was evident. (D) Nine hours after SMA ligation, the ischemic small bowel became black in color due to hemorrhage in the infracted bowel. 
Table I. The mean levels of serum enzymes in the mesenteric artery ligation group (IU).

\begin{tabular}{|c|c|c|c|c|c|c|c|c|}
\hline (h) & ALT & AST & ALP & $\gamma$-GT & CK & HBD & LDH & CK-MB \\
\hline 0 & $79.8 \pm 8.6$ & $90.7 \pm 7.4$ & $89.4 \pm 6.4$ & $10.3 \pm 0.6$ & $5984.3 \pm 424.9$ & $658.4 \pm 44.1$ & $686.3 \pm 32.3$ & $9302.0 \pm 670.0$ \\
\hline 1 & $89.1 \pm 6.3$ & $59.9 \pm 3.0$ & $73.8 \pm 4.3$ & $10.4 \pm 0.9$ & $5370.3 \pm 290.0$ & $421.8 \pm 25.7$ & $424.3 \pm 21.6$ & $8339.6 \pm 533.7$ \\
\hline 3 & $85.7 \pm 7.1$ & $82.3 \pm 5.3$ & $90.0 \pm 5.5$ & $8.2 \pm 0.6$ & $5824.5 \pm 366.9$ & $542.8 \pm 26.6$ & $560.2 \pm 33.6$ & $9374.3 \pm 684.3$ \\
\hline 6 & $117.8 \pm 12.0^{\mathrm{a}}$ & $133.8 \pm 9.2$ & $96.6 \pm 4.5$ & $10.3 \pm 0.6$ & $6883.8 \pm 399.3$ & $431.8 \pm 16.8$ & $522.3 \pm 32.4$ & $11169.4 \pm 882.4$ \\
\hline 9 & $148.8 \pm 8.9$ & $235.0 \pm 20.1^{\mathrm{b}}$ & $104.0 \pm 7.4$ & $5.7 \pm 0.5$ & $6372.8 \pm 312.3$ & $406.3 \pm 19.5$ & $572.8 \pm 28.1$ & $8789.4 \pm 571.3$ \\
\hline
\end{tabular}

${ }^{a}$ Compared to pre-ligation, $\mathrm{P}<0.05$; ${ }^{\mathrm{b}}$ compared to pre-ligation, $\mathrm{P}<0.01$. ALT, alanine aminotransferase; AST, aspartate aminotransferase; ALP, alkaline phosphatase; $\gamma$-GT, $\gamma$-glutamyl transpeptidase; CK, creatine kinase; HBD, hydroxybutyrate dehydrogenase; LDH, lactate dehydrogenase; CK-MB, creatine kinase-MB.

transferase (ALT), alkaline phosphatase (ALP), $\gamma$-glutamyl transpeptidase $(\gamma-\mathrm{GT})$, creatine kinase $(\mathrm{CK})$, creatine kinaseMB (CK-MB) and lactate dehydrogenase, (LDH) levels were measured in a Technicon RA-XT autoanalyzer using commercially available spectrophotometric kits (7).

Data analysis. The results were presented as the means \pm SD and used for statistical analysis using t-tests. A P-value of $<0.05$ was considered statistically significant.

\section{Results}

All control animals were found to have a normal-appearing bowel, while all the SMA or/and SMV ligation animals had an obvious abnormal-appearing bowel. The bowel became pale and changed to a grey color 5 to $10 \mathrm{~min}$ after vessel ligation. Three hours after ligation, focal necrosis of the small bowel was evident. Six hours after ligation, the animals had extensive patchy necrosis and dilation throughout the small bowel with free hemorrhagic intraperitoneal fluid. Nine hours after ligation, the small bowel was completely dilated and had changed to a black color. Most of the animals died $9 \mathrm{~h}$ after vessels ligation (Figs. 2 and 3).

In group I all the animals survived. In group II all the animals survived for up to $9 \mathrm{~h}$. The plasma enzyme levels are shown in Table I. Six and $9 \mathrm{~h}$ after SMA ischemia, ALT and AST levels reached 117 and 235 IU, respectively. Significant differences were found between pre-ligation and $6(\mathrm{P}<0.05)$ and $9 \mathrm{~h}$ after ligation $(\mathrm{P}<0.01)$ (Fig. 4). In group III, all the animals died within $6 \mathrm{~h}$. The plasma enzyme levels are shown in Table II. Three hours after SMV ligation, $\gamma$-GT levels in the blood reached $12 \mathrm{IU}$, which was considerably higher compared to the controls (mean value, 5.5 IU) (Fig. 5A). Six hours after SMV ischemia, ALT, AST, ALP and LDH levels were significantly elevated compared to those pre-ligation $(\mathrm{P}<0.05$ and 0.01) (Fig. 5B and C). In group IV, the plasma enzyme levels increased after vessel ligation, but no significant difference was observed (Table III).

\section{Discussion}

AMI is a potentially fatal vascular emergency which comprises a group of pathophysiological processes that have a common end-point, bowel necrosis. In addition, AMI can be charac-

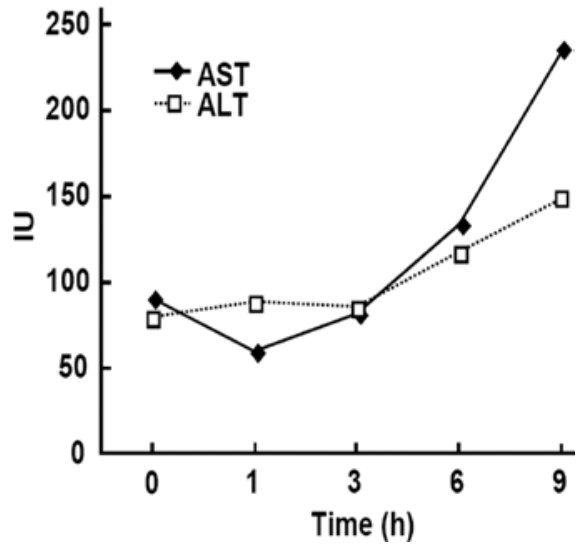

Figure 4. The serum alanine aminotransferase (ALT) and aspartate aminotransferase (AST) levels in the superior mesenteric artery (SMA) ligation group.

terized by an initial discrepancy between severe abdominal pain and minimal clinical findings. Moreover, many of the patients who develop AMI suffer from other severe medical conditions, such as peritonitis, ileus, pancreatitis and gastrointestinal bleeding at the same time, which make the symptoms less reliable $(8,9)$. Due to the non-specific clinical symptoms and presentations, early diagnosis and intervention of AMI are remain very difficult.

Previous studies have attempted to find simple laboratory tests which might identify AMI before bowel infarction and irreversible changes have occurred. Most of these studies have focused on potential biological markers of gut ischemia and injury, including measuring various intracellular enzymes released by the ischemic bowel, such as aminotransferase, LDH, D(-)-lactate, tyrosin, creatine phosphokinase and alcohol dehydrogenase (10-14). Unfortunately, all these markers have a low specificity and can not be used in clinical practice.

In other similar studies, serum AST, ALP, LDH and CK levels were investigated in order to determine intestinal necrosis resulting from ischemia $(15,16)$. In those studies, significant increases in peripheral enzyme levels were generally found after $\geq 3 \mathrm{~h}$ periods of ischemia. In this study, we found that ALT and AST levels increased after $>6 \mathrm{~h}$ in the SMA ligation group, while ALT, AST, ALP and LDH levels increased after $6 \mathrm{~h}$ in the SMV ligation group. Although ALP is considered as a diagnostic parameter of ischemic bowel, it was not found 
Table II. The mean levels of serum enzymes in the mesenteric vein ligation group (IU).

\begin{tabular}{lcccccccc}
\hline (h) & ALT & AST & ALP & $\gamma$-GT & CK & HBD & LDH & CK-MB \\
\hline 0 & $51.3 \pm 2.4$ & $38.5 \pm 2.3$ & $82.3 \pm 3.9$ & $5.5 \pm 0.3$ & $1389.7 \pm 116.7$ & $691.8 \pm 51.2$ & $673.0 \pm 56.5$ & $2649.3 \pm 190.7$ \\
1 & $50.8 \pm 2.8$ & $117.0 \pm 7.9$ & $37.8 \pm 2.5$ & $5.5 \pm 0.3$ & $1000.3 \pm 58.0$ & $309.2 \pm 14.8$ & $422.8 \pm 27.3$ & $1877.4 \pm 146.4$ \\
3 & $82.5 \pm 5.0$ & $211.0 \pm 15.4$ & $198.5 \pm 8.9$ & $12.0 \pm 0.8^{\mathrm{a}}$ & $1108.5 \pm 104.2$ & $297.0 \pm 24.7$ & $636.0 \pm 36.9$ & $1731.0 \pm 155.7$ \\
6 & $232.0 \pm 17.6^{\mathrm{b}}$ & $1208.0 \pm 108.7^{\mathrm{a}}$ & $248.0 \pm 18.6^{\mathrm{b}}$ & $28.0 \pm 0.5$ & $1937.0 \pm 166.6$ & $737.0 \pm 50.1$ & $1792.0 \pm 145.2^{\mathrm{b}}$ & $8638.6 \pm 682.4$ \\
\hline
\end{tabular}

${ }^{\mathrm{a} C}$ Compared to pre-ligation, $\mathrm{P}<0.05$; ${ }^{\mathrm{b}}$ compared to pre-ligation, $\mathrm{P}<0.01$. ALT, alanine aminotransferase; AST, aspartate aminotransferase; ALP, alkaline phosphatase; $\gamma$-GT, $\gamma$-glutamyl transpeptidase; CK, creatine kinase; HBD, hydroxybutyrate dehydrogenase; LDH, lactate dehydrogenase; $\mathrm{CK}-\mathrm{MB}$, creatine kinase-MB.

Table III. The mean levels of the serum enzymes in the group with both mesenteric artery and vein ligation (IU).

\begin{tabular}{lcccccccc}
\hline (h) & ALT & \multicolumn{1}{c}{ AST } & ALP & \multicolumn{1}{c}{$\gamma$-GT } & CK & HBD & LDH & CK-MB \\
\hline 0 & $83.5 \pm 5.6$ & $71.5 \pm 3.1$ & $163.0 \pm 11.6$ & $9.0 \pm 0.4$ & $4971.3 \pm 427.5$ & $896.5 \pm 51.1$ & $844.3 \pm 41.3$ & $8118.0 \pm 617.0$ \\
1 & $86.0 \pm 6.1$ & $90.0 \pm 4.1$ & $166.0 \pm 12.9$ & $10.0 \pm 0.6$ & $5300.0 \pm 376.3$ & $700.0 \pm 34.3$ & $870.0 \pm 79.1$ & $6701.0 \pm 583.0$ \\
3 & $93.8 \pm 7.7$ & $122.0 \pm 9.3$ & $150.5 \pm 10.7$ & $12.3 \pm 1.0$ & $3636.5 \pm 243.6$ & $279.5 \pm 13.1$ & $362.0 \pm 29.3$ & $5993.8 \pm 533.4$ \\
6 & $132.8 \pm 12.5$ & $280.3 \pm 20.7$ & $135.8 \pm 8.4$ & $10.5 \pm 0.7$ & $4910.8 \pm 309.4$ & $482.8 \pm 29.9$ & $672.0 \pm 45.7$ & $8101.8 \pm 575.2$ \\
9 & $267.3 \pm 18.0$ & $744.0 \pm 51.3$ & $123.0 \pm 10.7$ & $11.7 \pm 1.0$ & $6373.7 \pm 363.3$ & $839.0 \pm 59.6$ & $1196.7 \pm 96.9$ & $10431.7 \pm 777.9$ \\
\hline
\end{tabular}

ALT, alanine aminotransferase; AST, aspartate aminotransferase; ALP, alkaline phosphatase; $\gamma$-GT, $\gamma$-glutamyl transpeptidase; CK, creatine kinase; HBD, hydroxybutyrate dehydrogenase; LDH, lactate dehydrogenase; CK-MB, creatine kinase-MB.
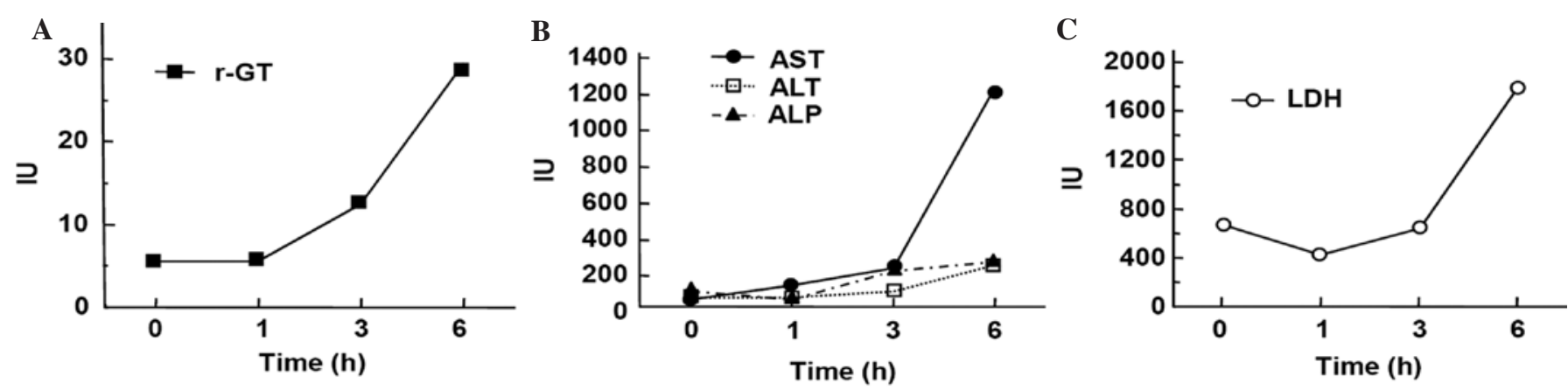

Figure 5. Serum $\gamma$-glutamyl transpeptidase $(\gamma$-GT), alanine aminotransferase (ALT), aspartate aminotransferase (AST), alkaline phosphatase (ALP) and lactate dehydrogenase (LDH) levels in the superior mesenteric venous (SMV) ligation group. (A) $\gamma$-GT level in the SMV ligation group. (B) Serum ALT, AST and ALP levels in the SMV ligation group. (C) Serum LDH changes in the animals with SMV ligation.

to increase earlier. CK-MB isoenzyme has been reported to be a more sensitive indicator of bowel necrosis than the intestinal isoenzyme of ALP (13). However, increases of CK and CK-MB were not found at any stage in our study. Additionally, a significant increase in $\gamma$-GT levels was also found after $3 \mathrm{~h}$ of ischemia, earlier than other enzymes, in the SMV ligation rabbits. We presume that the increase in $\gamma$-GT levels may be caused by hypoperfusion and injury of the liver.

There are several reasons why our results differ from those of previous studies. Firstly, complete or incomplete ischemia and the presence of venous or lymphatic drainage from the damaged tissue may affect blood enzyme levels during ischemia. Secondly, due to the massive metabolic capacity of the liver, the concentrations of these substances in the peripheral blood remain fairly stable and unchanged unless serious or irrevers- ible damage has occurred. In addition, different experimental animals used in these studies may also play a role.

Hepatic hypoperfusion and acute hepatic damage after ischemia were also reported. It was shown that after $2 \mathrm{~h}$ of intestinal ischemia, the ALT level increased up to four times the normal level (17). In this study, plasma ALT, AST and $\gamma$-GT levels increased significantly in the SMV ligation models, possibly due to hepatic hypoperfusion and damage to the liver during the study period.

In conclusion, as has also previously been shown, the increased enzyme levels cannot provide sufficient information as regards the extent and reversibility of intestinal injury, but only to reflect the presence of the injury (18). Increase in serum enzyme levels during intestinal ischemia may also depend on the animal models used. The significant increases in serum 
enzyme levels are usually late signs and are frequently associated with bowel infarction. Thus, early diagnosis of AMI can only be made by a physical examination, clinical presentation and laboratory tests, using the help of modern diagnostic techniques, such as CT, MRI and angiography.

\section{References}

1. Oldenburg WA, Lau LL, Rodenberg TJ, Edmonds HJ and Burger CD: Acute mesenteric ischemia: a clinical review. Arch Intern Med 164: 1054-1062, 2004.

2. Yasuhara H: Acute mesenteric ischemia: the challenge of gastroenterology. Surg Today 35: 185-195, 2005.

3. John BC and Theodore AS: Mesenteric ischemia: acute and chronic. Ann Vasc Surg 17: 323-328, 2003.

4. Brandt LJ and Boley SJ: AGA technical review on intestinal ischemia. American Gastrointestinal Association. Gastroenterology 118: 954-968, 2000.

5. Schoots IG, Koffeman GI, Legemate DA, Levi $M$ and van Gulik TM: Systematic review of survival after acute mesenteric ischaemia according to disease aetiology. Br J Surg 91: 17-27, 2004.

6. Bradbury AW, Brittenden J, McBride K and Ruckley CV: Mesenteric ischaemia: a multidisciplinary approach. Br J Surg 82: 1446-1459, 1995.

7. Que YH, Kaneko J, Sugawara Y, Tamura S and Makuuchi M: Role of protocol ultrasonography for detecting biliary stricture in adult living donor liver transplantation recipients. Biosci Trends 1: 62-65, 2007.

8. Park WM, Gloviczki P, Cherry KJ Jr, et al: Contemporary management of acute mesenteric ischemia: factors associated with survival. J Vasc Surg 35: 445-452, 2002.
9. Sreenarasimhaiah J: Diagnosis and management of intestinal ischaemic disorders. BMJ 326: 1372-1376, 2003.

10. Caglayan F, Caglayan O, Gunel E, Elcuman Y and Cakmak M: Intestinal ischemia-reperfusion and plasma enzyme levels. Pediatr Surg Int 18: 255-257, 2002.

11. Murray MJ, Barbose JF and Cobb CF: Serum D(-)-lactate levels as a predictor of acute intestinal ischemia in a rat model. J Surg Res 54: 507-509, 1993.

12. Contrin LM, Lobo SM, Navegantes LC, et al: Tyrosin: a possible marker of severe intestinal injury during iachemia. J Surg Res 155: 268-272, 2009.

13. Smirniotis VE, Labrou AT and Tsiftses DD: Plasma level of the creatine phosphokinase $\mathrm{BB}$ isoenzyme during experimental intestinal ischemia. Ann Vasc Surg 3: 8-10, 1989.

14. Gumaste UR, Joshi MM, Mourya DT, Barde PV, Shrivastav GK and Ghole VS: Alcohol dehydrogenase: a potential new marker for diagnosis of intestinal ischemia using rat as a model. World $\mathrm{J}$ Gastroenterol 11: 912-916, 2005.

15. De Toma G, Marzano D, Salvatore P, et al: Enzymatic and metabolic changes in peripheral serum after superior mesenteric artery ligation in dogs. Ital J Surg Sci 13: 269-273, 1983.

16. Graeber GM, Wolf RE and Harmon JW: Serum creatine kinase and alkaline phosphatase in experimental small bowel infarction. J Surg Res 37: 25-32, 1984.

17. Turnage RH, Kadesky KM, Myers SI, Guice KS and Oldham KT: Hepatic hypoperfusion after intestinal reperfusion. Surgery 119: 151-160, 1996.

18. Thompson JB, Bragg LE and West WW: Serum enzyme levels during intestinal ischemia. Ann Surg 211: 369-373, 1990. 